4. - Permutable periodic transformations, Proc. Nat. Acad. Sci. U.S.A. 30 (1944), 105-108.

5. - New results and old problems in finite transformation groups, Bull. Amer. Math. Soc. 66 (1960), 401-415.

INSTITUTE FOR DEFENSE ANALYSES AND

UNIVERSITY OF VIRGINIA

\title{
DIFFERENTIABLE ACTIONS OF COMPACT ABELIAN LIE GROUPS ON $S^{n}$
}

\author{
L. N. MANN
}

1. Introduction. In [9] P. A. Smith raises the following question: If a finite group $G$ acts effectively on the $n$-sphere $S^{n}$, must there also be some effective orthogonal action of $G$ on $S^{n}$ ? Stated another way, must all finite groups acting effectively on $S^{n}$ be isomorphic to subgroups of the orthogonal group $O(n+1)$ ? Smith has answered this question in the affirmative for the case where $G$ is an elementary p-group [8], [9]. The Corollary to Theorem 2 of this paper settles the case where $G$ is a compact abelian Lie group (in particular, a finite abelian group) and the action is assumed differentiable.

The proof of our main result is immediate if one assumes the existence of a fixed point, as evidenced by the following result which utilizes Bochner's theorem on local linearity about a fixed point.

Theorem 1. Let $G$ be a compact Lie group operating effectively and differentiably on a differentiable $n$-manifold $X$. If there exits a point $x_{0}$ left fixed by every element of $G$, then $G$ is isomorphic to a subgroup of $O(n)$.

Proof. By Bochner's theorem [5, p. 206], we may assume $G$ acts orthogonally (but not necessarily effectively) on some small closed $n$ cell $D$ with center $x_{0} . G$ leaves bdy $D=S^{n-1}$ invariant. If $G$ is not effective on $S^{n-1}$, then there must be a homeomorphism $g_{0}$ of finite order in $G$ which leaves $S^{n-1}$ pointwise fixed. Since $g_{0}$ acts linearly on $D$ and leaves $x_{0}$ fixed, it must also leave $D$ pointwise fixed. By Newman's theorem [5, p. 223], $g_{0}$ must leave $X$ pointwise fixed, violating the effectiveness of $G$ on $X$. Hence $G$ acts orthogonally and effectively on $S^{n-1}$, and the theorem follows.

Received by the editors March 10, 1964. 
2. Definitions and preliminaries. An action of a transformation group $G$ on a space $X$ is said to be effective if $g x=x$ for all $x$ in $X$ implies $g=e$, the identity element of $G$. All spaces considered will be compact Hausdorff spaces and the usual Cech cohomology will be used. Let $Z_{p}$ denote the additive group of integers modulo a prime $p$. Our definition of a cohomology n-manifold over $Z_{p}$ and of cohomology dimension over $Z_{p}$ will be that given in [1]. An elementary $p$-group of rank $k$ is a group isomorphic to the direct sum of $k$ copies of $Z_{p}$. Smith [7] has shown that if an elementary p-group $G$ acts effectively on a connected cohomology $n$-manifold $X$ over $Z_{p}$, then each component of $F(G, X)$, the fixed-point set of $G$ on $X$, is a connected cohomology $r$-manifold over $Z_{p}$ with $r<n$ for each $r$. In fact, if $p$ is an odd prime, each $n-r$ must be even. A generalized cohomology $n$ sphere over $Z_{p}$ is a cohomology $n$-manifold over $Z_{p}$ which has the global cohomology, coefficient group $Z_{p}$, of $S^{n}$. Results of Smith [6], [7] show that if an elementary p-group $G$ acts effectively on a generalized cohomology $n$-sphere $X$ over $Z_{p}$, then $F(G, X)$ is a generalized cohomology $r$-sphere over $Z_{p}, r<n$. We shall need the following result from $[3]$ :

LEMMA 1. Let $G$ be an elementary p-group of rank $k$ acting effectively on a connected n-manifold $X$. Suppose $F(G, X)$ is nonempty and of cohomology dimension $r$. Then

$$
k \leqq \begin{cases}\frac{n-r}{2} & \text { for } p \neq 2, \\ n-r & \text { for } p=2 .\end{cases}
$$

\section{Main results.}

ThEOREM 2. Let $G$ be isomorphic to the direct sum of $k$ copies of $Z_{2}$ and $l$ copies of $Z_{2 p}$, $p$ an odd prime. If $G$ acts effectively and differentiably on $S^{n}$, then

$$
k+2 l \leqq n+1 \text {. }
$$

Proof. By the above-mentioned Smith result [8], [9], we may assume $k, l \geqq 1$. We shall suppose $k+2 l=n+2$ and arrive at a contradiction.

There exists a subgroup $H$ of $G$ which is an elementary 2-group of rank $k+l$. We consider the action of $H$ on $S^{n}$. As usual, the isotropy subgroup of $H$ at a point $x$ in $X$ is defined as the subgroup of $H$ consisting of all elements which leave $x$ fixed. By the results of [8] or [4], there must exist an isotropy subgroup $H_{1}$ of $H$ of rank $k+l-1$. Since $H_{1}$ is an isotropy subgroup, $F(H, X)$ must be a proper subset 
of $F\left(H_{1}, X\right)$. Proceeding inductively, we may construct the following two decompositions

$$
\begin{aligned}
& H=H_{0} \supset H_{1} \supset \cdots \supset H_{k+l-1} \supset H_{k+l}=e \\
& \varnothing \subseteq F_{0} \subset F_{1} \subset \cdots \subset F_{k+l-1} \subset F_{k+l}=S^{n}
\end{aligned}
$$

where

(i) $H_{i}, 0 \leqq i \leqq k+l$, is a subgroup of $H$ of rank $k+l-i$ which operates on $S^{n}$ with fixed-point set $F_{i}$.

(ii) Each $F_{i}$ is a generalized cohomology sphere over $Z_{2}$.

(iii) Each $F_{i}$ is a compact manifold. This fact follows from the differentiability of the action by applying Bochner's theorem. Moreover, by (ii), each $F_{i}$ of positive dimension is connected.

(iv) $\operatorname{dim} F_{i}<\operatorname{dim} F_{i+1}, 0 \leqq i \leqq k+l-1$.

We proceed to investigate the decomposition (2) of $S^{n}$. From $\varnothing$ to $S^{n}$, there are exactly $(n+2)-(k+l+1)=l-1$ gaps in dimension, that is, dimensions not assumed by the $F_{i}$ 's. It follows that $\operatorname{dim} F_{i_{0}}$ is even (including 0 ) for some $i_{0}$, with $\operatorname{dim} F_{i_{0}} \leqq 2 l-2$; for, otherwise, there would have to be at least $l$ gaps. Select then the first $i_{0}$ with $\operatorname{dim} F_{i_{0}}$ even. As $k \geqq 1$, we have $\operatorname{dim} F_{i_{0}} \leqq 2 l-2<n$.

There exists a subgroup $K$ of $G$ which is an elementary p-group of rank $l$. The action of $K$ on $S^{n}$ leaves $F_{i_{0}}$ invariant. Since $F_{i_{0}}$ is a generalized cohomology sphere over $Z_{2}$, the Euler characteristic, $\chi\left(F_{i_{0}}\right)$, of $F_{i_{0}}$, which is independent of the coefficient field, must be equal to 2 . Hence, $\chi\left(F_{i_{0}}\right)$ is not congruent to 0 modulo $p$, and by an easy extension of Floyd's result in [2] to actions of elementary $p$-groups, the action of $K$ on $F_{i_{0}}$ must have a fixed point. By Lemma 1, there exists a subgroup $T$ of $K$ such that each element of $T$ leaves $F_{i_{0}}$ pointwise fixed and such that $K / T$ is effective on $F_{i_{0}}$ with

$$
\Gamma(K / T)=\operatorname{rank}(K / T) \leqq \frac{\operatorname{dim} F_{i_{0}}}{2}
$$

We shall say that $T$ is completely noneffective on $F_{i_{0}}$. Now

$$
\Gamma(T) \geqq \Gamma(K)-\frac{\operatorname{dim} F_{i_{0}}}{2}=l-\frac{\operatorname{dim} F_{i_{0}}}{2} \geqq l-\frac{2 l-2}{2}=1 .
$$

We next construct a decomposition of $T$,

$$
T=T_{i_{0}} \supseteq T_{i_{0}+1} \supseteq \cdots \supseteq T_{k+l} \supset e,
$$

such that $T_{j}, i_{0} \leqq j \leqq k+l$, is completely noneffective on $F_{j}$. As $T_{k+l}$ is a nontrivial subgroup of $G$ which is noneffective on $F_{k+l}=S^{n}$, we obtain a contradiction to the effectiveness of $G$. 
We proceed with the construction of (3). Now at least $\left(\operatorname{dim} F_{i_{0}}\right) / 2$ gaps have been used in arriving to $F_{i_{0}}$ in (2). Therefore there exist at most

$$
(l-1)-\frac{\operatorname{dim} F_{i_{0}}}{2}
$$

gaps from $F_{i_{0}}$ to $F_{k+l}$ in (2). Let $N=\operatorname{dim} F_{i_{0}+1}-\operatorname{dim} F_{i_{0}}$ and consider the following two cases.

(i) $N=1$. Now $T$ leaves the compact connected manifold $F_{i_{0}+1}$ invariant with fixed-point set containing $F_{i_{0}}$. Since $N=1$ and each element of $T$ is of prime order $p, p$ odd, we have that $T$ is completely noneffective on $F_{i_{0}+1}$ due to above-mentioned parity restrictions. In this case, choose $T_{i_{0}+1}=T$.

(ii) $N \geqq 2$. In this case, there are $N-1$ gaps from $F_{i_{0}}$ to $F_{i_{0}+1}$ in (2). Again, $T$ leaves $F_{i_{0}+1}$ invariant with fixed-point set $F$ containing $F_{i_{0}}$. By Lemma 1 , there exists a subgroup $T_{i_{0}+1}$ of $T$ which is completely noneffective on $F_{i_{0}+1}$ with

$$
\begin{aligned}
\Gamma\left(T / T_{i_{0}+1}\right) & \leqq \frac{\operatorname{dim} F_{i_{0}+1}-\operatorname{dim} F}{2} \\
& \leqq \frac{\operatorname{dim} F_{i_{0}+1}-\operatorname{dim} F_{i_{0}}}{2}=\frac{N}{2} .
\end{aligned}
$$

Consequently,

$$
\Gamma\left(T_{i_{0}+1}\right) \geqq \Gamma(T)-\frac{N}{2} \geqq \Gamma(T)-(N-1) .
$$

We see that each gap results in reducing the rank of $T$ to that of $T_{i_{0}+1}$ by at most one.

Proceeding inductively, and recalling that there are at most

$$
(l-1)-\frac{\operatorname{dim} F_{i_{0}}}{2}
$$

gaps from $F_{i_{0}}$ to $F_{k+l}$, we obtain

$$
\begin{aligned}
\Gamma\left(T_{k+l}\right) & \geqq \Gamma(T)-\left[(l-1)-\frac{\operatorname{dim} F_{i_{0}}}{2}\right] \\
& \geqq\left[l-\frac{\operatorname{dim} F_{i_{0}}}{2}\right]-\left[(l-1)-\frac{\operatorname{dim} F_{i_{0}}}{2}\right]=1 .
\end{aligned}
$$

COROLLARY. Let $G$ be a compact abelian Lie group acting effectively 
and differentiably on $S^{n}$. Then $G$ is isomorphic to a subgroup of $O(n+1)$.

Proof. $G$ is the direct sum of a $q$-torus $T^{q}$ and a finite abelian group $R$. Choose a minimal set of generators of $R$, let $g(R)$ denote the total number of these generators and $h(R)$ denote the number of these generators of order 2. Suppose, first, that $h(R)=0$. Then there exists for some odd prime $p$ a subgroup $G^{\prime}$ of $G$ with $G^{\prime}$ an elementary p-group of rank $q+g(R)$. Then, by Smith [8], [9],

$$
q+g(R) \leqq\left[\frac{n+1}{2}\right]
$$

and the Corollary follows. Suppose then that $h(R) \geqq 1$. Then there exists a subgroup $G^{\prime \prime}$ of $G$ with $G^{\prime \prime}$ isomorphic to the direct sum of $h(R)$ copies of $Z_{2}$ and $[q+g(R)-h(R)]$ copies of $Z_{2 p}$, for some odd prime $p$. The Corollary now follows from Theorem 2 .

\section{REFERENCES}

1. A. Borel et al., Seminar on transformation groups, Princeton Univ. Press, Princeton, N. J., 1960.

2. E. E. Floyd, On periodic maps and the Euler characteristics of associated spaces, Trans. Amer. Math. Soc. 72 (1952), 138-147.

3. L. N. Mann and J. C. Su, Actions of elementary p-groups on manifolds, Trans. Amer. Math. Soc. 106 (1963), 115-126.

4. L. N. Mann, $A$ note on counting isotropy subgroups, Proc. Amer. Math. Soc. 16 (1965) 476-480.

5. D. Montgomery and L. Zippin, Topological transformation groups, Interscience, New York, 1955.

6. P. A. Smith, Transformations of finite period, Ann. of Math. (2) 39 (1938), 127-164.

7. - Transformations of finite period. II, Ann. of Math. (2) 40 (1939), 690-711.

8. - Permutable periodic transformations, Proc. Nat. Acad. Sci. U.S.A. 30 (1944), 105-108.

9. - New results and old problems in finite transformation groups, Bull. Amer. Math. Soc. 66 (1960), 401-415.

Institute For Defense Analyses AND UNIVERSITY OF VIRGINIA 